\title{
Effects of Sodium Chloride on Tyramine Production in a Fermented Food Model and its Inhibition by Tyrosine-degrading Lactobacillus plantarum JA-1199
}

\author{
Janine Anderegg, Florentin Constancias, and Leo Meile*
}

\begin{abstract}
Tyramine is a health-adverse biogenic amine, which can accumulate in fermented foods like cheese by decarboxylation of the free amino acid tyrosine by either starter cultures or resident microbes such as lactic acid bacteria including Enterococcus spp., respectively. Our study aimed to show the effect of sodium chloride concentrations on tyramine production as well as to characterise bacterial strains as anti-tyramine biocontrol agents in a $2 \mathrm{~mL}$ micro-cheese fermentation model. The effect of sodium chloride on tyramine production was assayed with tyramine producing strains from eight different species or subspecies. Generally, an increase in sodium chloride concentration enhanced tyramine production, e.g. from $0 \%$ to $1.5 \%$ of sodium chloride resulted in an increase of tyramine of $870 \%$ with a Staphylococcus xylosus strain. In the biocontrol screening among lactic acid bacteria, a Lactobacillus plantarum JA-1199 strain was screened that could consume in successful competition with other resident bacteria tyrosine in the micro-cheese model as a source of energy gain. Thereby tyramine accumulation was reduced between $4 \%$ to $99 \%$. The results of this study disclose a feasible strategy for decreasing tyramine concentration and increasing the safety level of fermented food. It is an example of development and application of bacterial isolates as starter or protective cultures in food, a biocontrol topic, which Oreste Ghisalba - in his project evaluation function of SNF and later on CTI - was promoting with great emphasis in our ETH Food Biotechnology research group.
\end{abstract}

Keywords: Biocontrol · Dairy products · Degrading lactic acid bacteria · Tyramine $\cdot$ Tyrosine

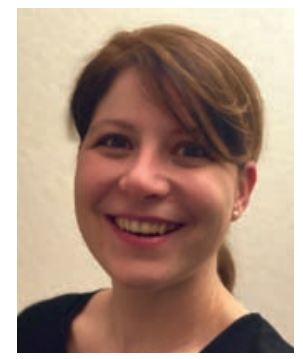

Janine Anderegg studied Food Science and completed her MSc in Food Science with a focus on Food Quality and Safety at ETH Zurich. In 2016 she moved to the Food Biotechnology group of Prof. Christophe Lacroix, at ETH Zurich to pursue her PhD in microbiology and molecular biology with the main focus on the role of the microbial community in the production of biogenic amines in fermented food products.

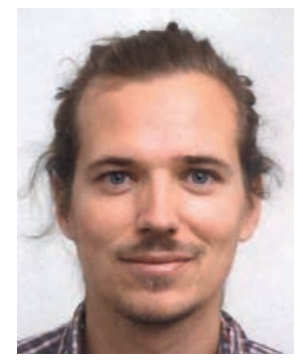

Florentin Constancias is a computational microbial ecologist fascinated by the diversity of microorganisms and their contribution in ecosystem functioning. He studied soil microbial ecology and developed expertise in bioinformatics. After his $\mathrm{PhD}$, he moved to NTU Singapore, where he applied metagenomics to address fundamental and applied questions such as assembly of bacterial communities or the impact of salivary microbiome in oral health and disease. He is currently a research assistant in the Food Biotechnology group at the ETH Zürich and focuses on gut microbiome and food microbiology.

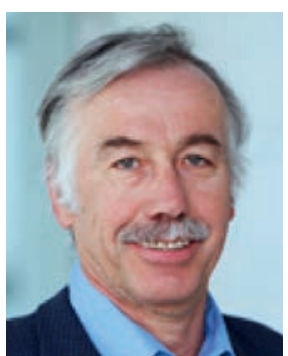

Leo Meile is a Titular Professor, recently emerited, at ETH Zurich in the Food Biotechnology group. The main focus of his research was on biodiversity of microorganisms in fermented food of industrial and developmental countries, their traits and functions in specialized ecotopes and microbiota, mechanisms of antimicrobial resistance, the development of new protective microbial cultures for biopreservation of food and starter culture development. His research combines modern knowledge of dominant microbes at genome level in fermented food from typical Swiss and developmental country products with new microbial strain innovations for food processing in order to achieve high food safety for consumers.

\section{Introduction}

Biogenic amines (BAs) are low-molecular weight organic bases formed by decarboxylation of free amino acids. According to their chemical structure, they can be classified as aliphatic (cadaverine, putrescine, spermine and spermidine), as aromatic (tyramine and phenylethylamine) or as heterocyclic (histamine and tryptamine). ${ }^{[1]}$ The most well known BAs are cadaverine, histamine, putrescine and tyramine derived from decarboxylation of the free precursor amino acids lysine, histidine, ornithine and tyrosine, respectively. ${ }^{[2,3]}$ BAs have been reported in a variety of foods, including dairy products, ${ }^{[1]}$ fermented vegetables ${ }^{[4]}$ and fruits, ${ }^{[5]}$ and also in fermented meat and fish. ${ }^{[6,7]}$ Although BAs can have important physiological functions in plants, animals and humans, ${ }^{[8-11]}$ the consumption of food with high concentrations of BAs can thereby pose a risk of food intoxication. ${ }^{[12]}$ For example, histamine and tyramine can cause severe allergic reactions such as 
nausea and headaches; increase respiration, cardiac output, blood glucose level and high blood pressure; and release norepinephrine. ${ }^{[13-15]}$ Compared to histamine and tyramine, cadaverine and putrescine have a much lower toxicological impact, but they are able to increase the toxic effect of other amines. ${ }^{[16]}$ Even though BAs can induce reverse reactions, only histamine in fishery products is covered by specific legislation for BAs. ${ }^{[17]}$

Tyramine derives from decarboxylation of the free amino acid tyrosine and is one of the most abundant, frequent BAs in food, especially in cheese and fermented sausages. ${ }^{[1,18,19]}$ There is currently insufficient information for tyramine to establish a no adverse health effect level (NOAEL) in humans. No adverse health effects have been observed in healthy individuals exposed to $600 \mathrm{mg}$ tyramine per person per meal. ${ }^{[15]}$ However, for individuals consuming alcohol or taking monoamine oxidase inhibitor drugs, the NOAEL is set much lower between 6 and $50 \mathrm{mg}$ of tyramine per person per meal.[12,15,20,21] Tyramine is produced by a variety of lactic acid bacteria (LAB) including carnobacteria, enterococci, lactobacilli and lactococci and by some staphylococci.[7,22,23] The key enzyme, a tyrosine decarboxylase (EC 4.1.1.25), together with a transporter protein for tyrosine/tyramine interchange, are involved in tyramine enrichment. ${ }^{[1,24,25]}$ All corresponding genes are clustered in an operon called $t d c$ or $t y r$, on the plasmids or chromosomes of tyramine producer strains.[26,27] The gene called $t d c A$ or $t d c$ encodes the tyrosine decarboxylase, while the gene called tyrP encodes the tyrosine/tyramine antiporter influx/efflux system. Tyrosine decarboxylase has been characterised extensively in a few LAB species, including Enterococcus faecalis, Enterococcus faecium, Lactobacillus brevis and Lactobacillus curvatus. ${ }^{[1,7]}$ The production of tyramine is possible only when at least three external factors converge. At first, the substrate amino acid tyrosine must be available; secondly, the presence of microorganisms with the appropriate catabolic pathway activated and thirdly, the environmental conditions must be favourable to the decarboxylation activity. ${ }^{[22]}$ The external factors can be influenced by $\mathrm{pH}$ and temperature of the environment, sodium chloride concentration in the media and availability of carbon sources. For example, a key factor for the production of tyramine is the tyrosinedecarboxylase enzyme, which has an optimum around pH 5.0 and also sodium chloride stress could upregulate the expression of the tyrosine decarboxylase and the tyrosine-tyramine antiporter genes. ${ }^{[2,28-32]}$

Traditional methods to prevent tyramine formation are primarily by limiting microbial growth through chilling and freezing. Further approaches to limit microbial growth and therefore, tyramine concentration include thermisation or pasteurisation, hydrostatic pressures, irradiation, controlled atmosphere packaging, or the use of food additives. ${ }^{[21,33-35]}$ Another technological measure to control tyramine during fermentation is the use of selected tyrosine decarboxylase negative starter cultures. $[18,36]$

A further approach, however rarely applied, for the reduction of tyramine is the use of selected bacterial strains provided with a specific amino oxidase, reducing in situ tyramine during food production. ${ }^{[37]}$ In the presence of oxygen, for example, such amino oxidases are able to deaminate BAs by the production of $\mathrm{NH}_{3}, \mathrm{H}_{2} \mathrm{O}_{2}$ and aldehydes. ${ }^{[38]}$ Enzymes providing this oxidase activity belong to the multicopper oxidase family (EC 1.10.3), a family of enzymes comprising oxidases such as ascorbate oxidase (EC 1.10.3.3), ferroxidases (EC 1.16.3.1), laccases (EC 1.10.3) as well as tyrosinase (EC 1.14.18.1). ${ }^{[39,40]}$ Multicopper oxidases are found in different bacterial species, and previous studies reported the application of Lactobacillus spp. containing a multicopper oxidase to decompose BAs. ${ }^{[41,42]}$ These aldehydes are further reduced to the corresponding acids, which can be transferred to the central metabolism of the cells. ${ }^{[43]}$ Another approach reducing tyramine in fermented food is the use of selected bacterial strains, which can use tyrosine as an energy source and thus act in compe- tition with tyramine-producing strains for the substrate tyrosine. Such enzymes are called aminotransferases, and they can catalyse the reversible pyridoxal phosphate-dependent transfer of amino groups from amino acids to oxo acids. ${ }^{[44]}$

The accumulation of tyramine and other BAs in fermented food products is not only a consequence of bacterial contaminants harbouring decarboxylase activity but also an uncontrolled metabolic activity of natural starter cultures, e.g. in traditionally produced cheese. ${ }^{[18,36]}$ The addition of a non-tyramine-producing but tyramine- or tyrosine-oxidising lactic acid bacterium as part of starter cultures would be an interesting strategy to reduce the accumulation of tyramine in traditionally produced foods while preserving the sensory properties. ${ }^{[3,42]}$

In this context, starting from many lactic acid bacteria previously isolated from food and screened for amino acid oxidising ability, this study aimed to explore whether a selected non-tyramine-producing lactic acid bacterium strain, is able to (1) use tyrosine to deplete this substrate for potential tyramine production or (2) to oxidise tyramine. Furthermore, this study aimed to identify the responsible enzyme in silico and to apply the selected strain in a micro-cheese model containing tyrosine and different sodium chloride concentration in combination with different tyramineproducing microorganism.

\section{Materials and Methods}

\subsection{Strains}

Lactobacillus plantarum JA-1199, previously isolated from a fermented sausage sample, was used in this study as a tyrosine degrader. $L b$. plantarum JA-1199 was routinely propagated anaerobically at $37^{\circ} \mathrm{C}$ for $24 \mathrm{~h}$ in De Man-Rogosa-Sharpe (MRS) broth. In this study, strains of Enterococcus faecalis 201701784.1.K, Enterococcus faecium VF21.2-1122.3.K, Enterococcus durans 201702044.1.K, Staphylococcus equorum 201701784.3.B, Staphylococcus simulans 201702044.2.B, Staphylococcus xylosus 201702052.2.B, Lactococcus lactis P.6.2.M and Lactobacillus parabuchneri P.12.3.M, respectively, were used as tyramine producers. These strains were previously isolated from cheese or fermented sausages by our research group and tested genoand phenotypically for the presence of the $t d c$-gene and tyrosine decarboxylase activity, respectively. Enterococcus spp. and Staphylococcus spp. were incubated aerobically at $37^{\circ} \mathrm{C}$ in brainheart infusion (BHI) broth while Lactobacillus and Lactococcus spp. were incubated anaerobically at $37^{\circ} \mathrm{C}$ in MRS broth.

\subsection{In silico Screening of Lb. plantarum JA-1199}

\subsubsection{Tyrosine Decarboxylase Enzyme EC 4.1.1.25}

The absence of the tyrosine decarboxylase gene $(t d c A)$ in $L b$. plantarum JA-1199, was investigated using an in silico screening approach. The genome of Lb. plantarum JA-1199 was sequenced with Illumina MiSeq (pairwise reads of $150 \mathrm{bp}$ ) with a coverage of 30-fold. Genes were the identified using prokka v.1.12 (http://www.vicbioinformatics.com/software.prokka.shtml) and the absence of tyrosine decarboxylase was screened by aligning amino acids sequences of $L b$. plantarum JA-1199 against tyrosine decarboxylase enzyme EC 4.1.1.25 sequence in the GeneBank Genome Net (https://www.genome.jp/), UniProtKB (https://www.uniprot.org/help/uniprotkb), and Brenda enzymes (https://www.brenda-enzymes.info/index.php) databases with the BLAST program included CLC Genomics workbench (version 10.0.1) program.

\subsubsection{Identification of Tyrosine Degrading Enzymes of $L b$. plantarum JA-1199}

Genes were also annotated using prokka v.1.12 and pathway analysis was conducted using the KEGG pathway web services (https://www.genome.jp/kegg/kaas/), the Laccase Engineering 
Database (LccED https://lcced.biocatnet.de/) and the NCBI gene bank (https://www.ncbi.nlm.nih.gov/).

\subsection{Phenotypic Identification of Tyrosine Degradation 2.3.1 Analysis of Metabolites by Biolog}

Carbon and nitrogen metabolism of $\mathrm{Lb}$. plantarum JA-1199 were analysed by the Biolog microarray systems PM3B (Nitrogen Sources) and PM1 (Carbon Source), respectively (Biolog, 21124 Cabot Blvd., Hayward, CA 94545, United States of America). The 96-well plates were inoculated with $0.1 \mathrm{~mL}$ of the calculated concentration (turbidity of 75\%) of the overnight bacterial suspension of $L b$. plantarum JA-1199 with an IF-0a GN/GP base inoculating fluid and a tetrazolium redox dye mix according to the manual. Plates were analysed at $30{ }^{\circ} \mathrm{C}$ for 3 days every 15 min and data were analysed using the program 'DuctApe' (https://combogenomics.github.io/DuctApe/index.html).

\subsubsection{Milk and Minimal Chemically Defined Medium (mCDM)}

Lb. plantarum JA-1199 was phenotypically assayed for its capacity to degrade tyrosine upon cultivation in pasteurised skim milk and minimal chemically defined medium (mCDM). ${ }^{[37]}$ Slight modifications by adding sterile-filtered copper (II) chloride to a final concentration of $1 \mathrm{mM}$ solution after sterilisation of mCDM and by changing the glucose concentration to $5 \mathrm{~g} \mathrm{~L}^{-1}$ were done. The overnight pre-culture of $L b$. plantarum JA-1199 was centrifuged at 7'000 x g for $10 \mathrm{~min}$, washed with peptone solution $(9 \% \mathrm{NaCl}, 1 \%$ peptone from casein, $\mathrm{pH} 7)$ and inoculated at a calculated $0 D 600_{n m}$ value of 0.1 in milk or mCDM broth containing $4 \mathrm{mM}$ tyrosine (Sigma Aldrich, Buchs, Switzerland) as a substrate for potential tyrosine degrading bacteria. Afterwards, samples were incubated at $37{ }^{\circ} \mathrm{C}$ for $96 \mathrm{~h}$, and tyrosine and tyramine were measured at the time points of $0,4,6,24,48,72$, and $96 \mathrm{~h}$. Bacterial growth was monitored by plating serial dilutions on the corresponding semi-selective MRS agar-medium incubated anaerobically for $48 \mathrm{~h}$ at $37^{\circ} \mathrm{C}$.

\subsection{Experimental Micro-cheese Model Manufacture}

A micro-cheese model, containing $2 \mathrm{~mL}$ pasteurised skimmed milk, $1 \mathrm{mM} \mathrm{CaCl}_{2}$ and $0.02 \%$ Fromase $220 \mathrm{TL}$ rennet (Winkler AG, Konolfingen, Switzerland) was prepared. All tyramine producer strains were tested in these micro-cheese models in triplicate under four different sodium chloride concentrations $(0 \%$, $1.5 \%, 3.0 \%$ and $4.5 \%$ ) each with and without adding $2.5 \mathrm{mM}$ tyrosine, either the strains alone or combined with $L b$. plantarum JA-1199 to test its capacity to reduce tyramine accumulation in a cheese-like environment. Overnight cultures of the used strains were centrifuged at 7'000 $\mathrm{x}$ g for $10 \mathrm{~min}$ and resuspended at a calculated $\mathrm{OD}_{600}$ of 0.2 in pasteurised skimmed milk. The casein degradation step was started through the added $\mathrm{CaCl}_{2}$ and rennet followed by incubation at $30{ }^{\circ} \mathrm{C}$ for $1 \mathrm{~h}$. The curd was cut by using a sterile inoculation loop and incubated for $15 \mathrm{~min}$ at $37{ }^{\circ} \mathrm{C}$, after which cutting was repeated followed by incubation at $37{ }^{\circ} \mathrm{C}$ for $40 \mathrm{~min}$ again. To simulate the pressing process of the cheese, micro-cheese samples were centrifuged at 6'000 x g for $10 \mathrm{~min}$. The supernatant was used for tyrosine, tyramine and $\mathrm{pH}$ measurement, and the micro-cheese pellet was incubated at $30{ }^{\circ} \mathrm{C}$ for 7 days to simulate the ripening time. After the maturation step, the pellet was resuspended in $1.8 \mathrm{~mL}$ Milli-Q water and $0.1 \mathrm{~mL}$ were used for colony counting. Afterwards, the resuspension was centrifuged at $10^{\prime} 000 \mathrm{x} \mathrm{g}$ for $5 \mathrm{~min}$, and the supernatant was used for tyrosine, tyramine and $\mathrm{pH}$ measurement. Colony counting was performed by plating serial dilutions of the $0.1 \mathrm{~mL}$ portion on the corresponding semi-selective agar-media Baird-Parker agar (BP; Biolife, Milano, Italy) for Staphylococcus spp., KF Streptococcus agar (KFS, Becton Dickinson, Allschwil, Switzerland) for Enterococcus spp. and De Man, Rogosa and Sharpe agar (MRS, Biolife, Milano, Italy) for Lactobacillus and
Lactococcus spp. BP and KFS agar were incubated aerobically for $48 \mathrm{~h}$ at $37^{\circ} \mathrm{C}$ and $43^{\circ} \mathrm{C}$, respectively whereas MRS agar was incubated anaerobically for $48 \mathrm{~h}$ at $37^{\circ} \mathrm{C}$ in $2.5 \mathrm{~L}$ culture jars with AnaeroGen AN 25 sachets (Oxoid, Basingstoke, UK).

\subsection{Determination of Tyrosine and Tyramine}

For the quantitative determination of tyrosine and tyramine, ion chromatography (IC) was used ${ }^{[45]}$ using the IC apparatus Dionex ${ }^{\mathrm{TM}}$ ICS-5000+ Hybrid HPIC ${ }^{\mathrm{TM}}$ system and IonPac CG18, 2 × $50 \mathrm{~mm}$ as a precolumn and IonPac CS18, 2 × 250 $\mathrm{mm}$ as separation column, respectively (Thermo Fisher Scientific, Switzerland). The Dionex method was modified by changing the eluent gradient: 5-10 mM methanesulfonic acid (MSA) from 0-6 min, 10-20 mM from 6-16 min, and 20-45 mM from 16-20 min. The samples were used in a 1:10 dilution for determination of tyrosine and tyramine concentrations with a detection limit of $0.13 \mathrm{mg} \mathrm{L}^{-1}$ and $0.05 \mathrm{mg} \mathrm{L}^{-1}$, respectively.

\subsection{Statistical Analysis}

All samples were measured in triplicate, and all means of replications were first calculated prior to comparison. Samples with concentrations of tyramine or tyrosine below the detection limit were replaced with artificial values of 0.049 for the concentration of tyramine and 0.129 for the concentration of tyrosine, respectively, to allow statistical analysis. ${ }^{46]}$ Statistical analysis was performed by using a t-test including Shapiro-Wilk normality and Brown-Forsythe equal variance test $(P<0.05)$ in the statistic software SigmaPlot 13 (Systat Software Inc., San Jose, California, USA).

\section{Results}

\subsection{In silico Screening of Lb. plantarum JA-1199}

In a preliminary test, different $L b$. plantarum strains, previously isolated from cheese and sausage samples, were tested on the ability to degrade tyrosine. Because $L b$. plantarum JA-1199 showed the highest capacity to degrade tyrosine and was under phenotypic assay conditions unable to produce tyramine, this strain was selected for further experiments. Indeed, its genome analysis revealed a genome-size of $3.33 \mathrm{Mb}, \mathrm{a} \mathrm{G}+\mathrm{C}$ content of $44.3 \%$ and showed a high level of similarity to $L b$. plantarum WFCS1, which is one of the best characterised $L b$. plantarum strains, and both strains, either at the nucleotide or at the protein level, lack a tyrosine decarboxylase gene $(t d c A)$ or tyrosine decarboxylase enzyme (EC 4.1.1.25), respectively.

In the in silico screening of Lb. plantarum JA-1199 for a tyrosine degrading enzyme, a multicopper oxidase family protein of 501 as well as a histidinol-phosphate transaminase (EC 2.6.1.9) of 365 amino acid residues were found. The detected enzyme showed on the protein BLAST level a $100 \%$ identity and an E-value of 0.0 to Lactobacillus multicopper oxidase domain-containing proteins (WP_003641930) and to the histidinol-phosphate transaminase protein (WP_046947669.1), which confirmed, that the compared amino acid sequences have the same residues at the same positions in an alignment.

\subsection{Phenotypic Identification of Tyrosine Degradation}

For a phenotypic confirmation of the in silico screening of $L b$. plantarum JA-1199, an analysis of metabolites by a Biolog microarray assay was done. This assay seemed to have the capacity to test the $L b$. plantarum JA-1199 strain as tyrosine degrader. $L b$. plantarum JA-1199 was able to use tyrosine as a nitrogen source. Moreover, the ability of the strain to use tyramine as a carbon source was negative.

In a further preliminary trial and for a second phenotypic confirmation of the capacity of $L b$. plantarum JA-1199 to degrade tyrosine, a phenotypic assay in pasteurised skimmed milk and upon 
the cultivation in $\mathrm{mCDM}$, both containing tyrosine, was done. In addition, IC-mediated measurement of tyrosine and tyramine concentrations after growth in both $\mathrm{mCDM}$ and milk confirmed the ability of $L b$. plantarum JA-1199 to degrade tyrosine up to $54.68 \%$ and $40.72 \%$, respectively, whereas the tyramine concentration was below the detection limit or in a minimum concentration of $6.05 \mathrm{mg} \mathrm{L}^{-1}$, respectively (data not shown).

\subsection{Tyramine Formation and Tyrosine Degradation in a Micro-cheese Model with Different Sodium Chloride Concentrations}

To investigate tyrosine degradation and tyramine formation in an environment similar to cheese with different sodium chloride concentrations, a micro-cheese model was performed. Therefore, sodium chloride concentration in a micro-cheese fabrication process at an initially fixed concentration of tyrosine $(2.5 \mathrm{mM})$ and variable sodium chloride concentrations were tested. Indeed sodium chloride markedly influenced tyramine formation of all eight bacterial strains, all selected before as tyramine producers, belonging to the species E. faecalis, E. faecium, E. durans, S. equorum, S. simulans, S. xylosus, L. lactis, and L. parabuchneri, respectively (Table 1).

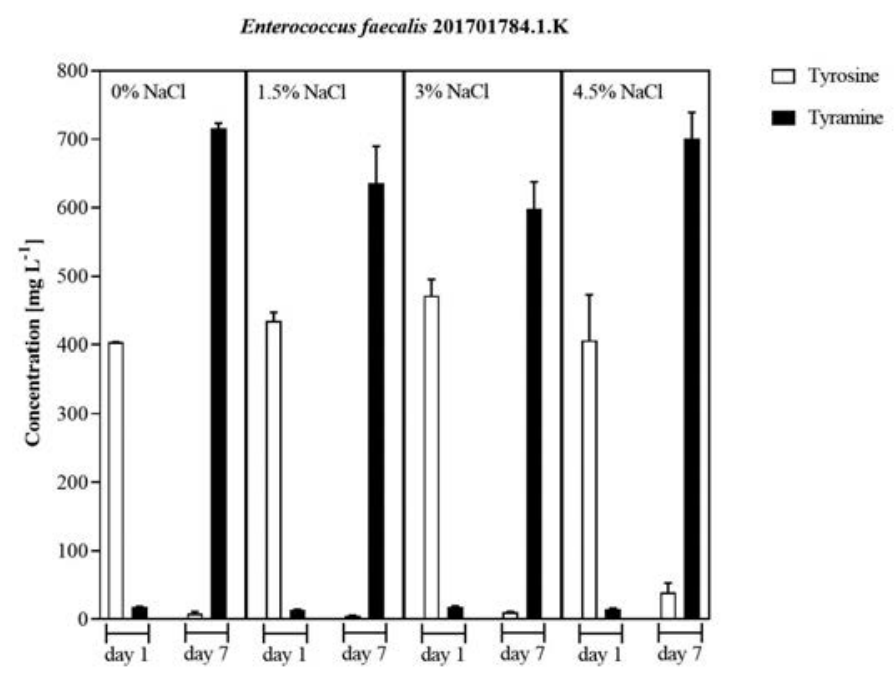

Fig. 1. Mean values of tyrosine (white) and tyramine (black) concentrations produced by $E$. faecalis $201701784.1 . \mathrm{K}$ at $0 \%, 1.5 \%, 3 \%$ and $4.5 \%$ sodium chloride incubated with $2.5 \mathrm{mM}$ tyrosine.

Table 1. Production of tyramine in $\mathrm{mg} \mathrm{L}^{-1}$ by eight different tyramine-producing bacterial strains in micro-cheese models containing $2.5 \mathrm{mM}$ tyrosine and four different sodium chloride concentrations.

Sodium chloride concentration

$\mathbf{0 \%}$

$1.5 \%$

$3 \%$

$4.5 \%$

Species

Enterococcus faecalis

201701784.1.K

VF21.2-1122.3.K

716.10

Tyramine production [mg L ${ }^{-1}$ ]

Enterococcus faecium

201702044.1.K

195.22

635.90

598.52

701.08

Enterococcus durans

201701784.3.B

301.15

325.75

566.20

653.26

Staphylococcus equorum

Staphylococcus simulans

201702044.2.B

Staphylococcus xylosus

201702052.2.B

107.92

248.16

342.42

685.32

72.12

575.93

613.11

600.66

349.82

143.73

337.65

67.91

659.17

322.92

573.75

Lactococcus lactis

P.6.2.M

Lactobacillus parabuchneri

P.12.3.M
264.96

262.71
349.32

417.40

517.15

369.25
429.85
Importantly, all of the eight strains were able to grow in a micro-cheese model containing up $4.5 \%$ of sodium chloride and initial tyrosine at $2.5 \mathrm{mM}$, and form concentrations of tyramine up to $701.08 \mathrm{mg} \mathrm{L}^{-1}$ in the cheese-model containing $E$. faecalis 201701784.1.K. In addition, this strain formed a relatively stable and high tyramine concentration in micro-cheeses at all four sodium chloride concentrations $(0 \%, 1.5 \%, 3 \%, 4.5 \%)$ (Fig. 1).

The highest increase of tyramine concentration was $870.72 \%$ and was produced by S. xylosus 201702052.2.B in the two microcheese models containing $0 \%$ and $1.5 \%$ sodium chloride and tyramine concentrations of $67.91 \mathrm{mg} \mathrm{L}^{-1}$ and $659.17 \mathrm{mg} \mathrm{L}^{-1}$, respectively (Table 1). All tyramine-producing bacteria, except $E$. faecalis 201701784.1.K, S. simulans 201702044.2.B and S. xylosus 201702052.2.B accumulated a higher concentration of tyramine in micro-cheese models at sodium chloride concentrations of 3 and $4.5 \%$ than at lower sodium chloride concentrations of 0 and $1.5 \%$, respectively. Furthermore, the three tyramine-producing bacteria L. lactis P.6.2.M, Lb. parabuchneri P.12.3.M, as well as E. faecium VF21.2-1122.3.K, showed with an increase of sodium chloride a continuous increase of tyramine concentration (Table1, Fig. 2).

In micro-cheeses started without the addition of tyrosine, tyramine concentrations were negligible during production and were

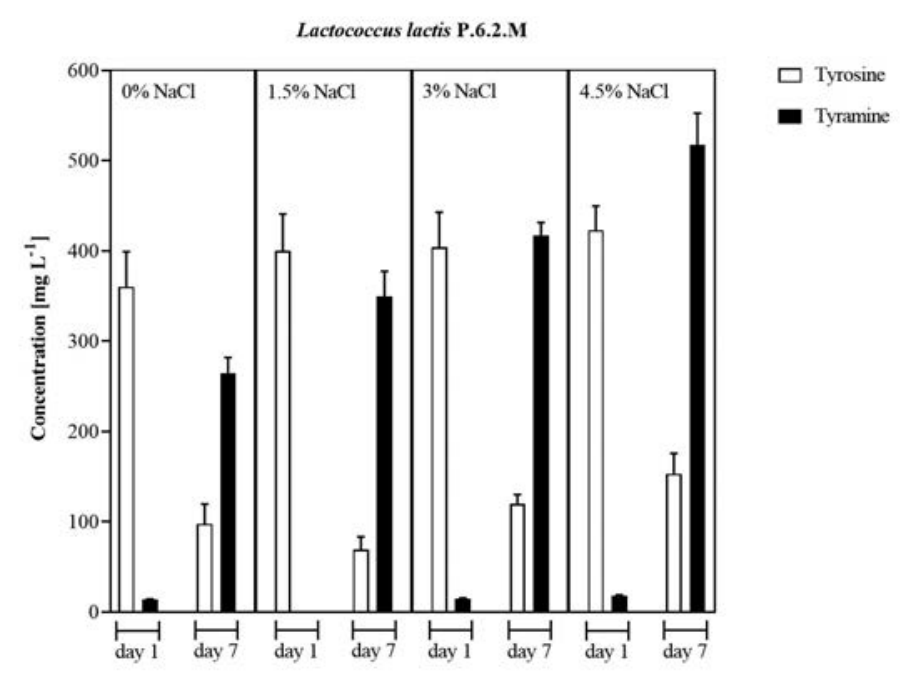

Fig. 2. Mean values of tyrosine (white) and tyramine (black) concentrations produced by L. lactis P.6.2.M at $0 \%, 1.5 \%, 3 \%$ and $4.5 \%$ sodium chloride incubated with $2.5 \mathrm{mM}$ tyrosine. 
between 'below detection limit' (S. equorum 201701784.3.B at $0 \%$ sodium chloride and $S$. simulans 201702044.2.B at 0 and $3 \%$ sodium chloride) and $17.55 \mathrm{mg} \mathrm{L}^{-1}$ (E. faecalis 201701784.1.K at $0 \%$ sodium chloride) (data not shown).

Interestingly, tyramine accumulation could be reduced in all setups containing one of the eight tyramine-producer strains by the addition of $L b$. plantarum JA-1199 except in the setup containing L. lactis P.6.2.M at 4.5\% sodium chloride. However, there were significant differences between different tyramine-producing strains as well as between the different sodium chloride concentrations within a tyramine producer itself (Table 2). with $3 \%$ sodium chloride, compared to the reduction in combination with other tested tyramine producers (Table 2).

In comparison, tyramine concentrations in micro-cheese models in combination with $L b$. plantarum JA-1199 without initial addition of $2.5 \mathrm{mM}$ tyrosine could be neglected since concentration reached only values between 'below the detection limit' (S. equorum 201701784.3.B, and S. xylosus 201702052.2.B at $0 \%$ sodium chloride and S. simulans 201702044.2.B at $0 \%, 1.5 \%$ and $3 \%$ sodium chloride) and $18.89 \mathrm{mg} \mathrm{L}^{-1}$ (E. faecalis $201701784.1 . \mathrm{K}$ at $0 \%$ sodium chloride) (Table 3 ).

Table 2. Reduction ability of tyramine-accumulation in percentage by Lb. plantarum JA-1199 in micro-cheese models containing 2.5 mM tyrosine and four different sodium chloride concentrations inoculated with eight different tyramine-producing strains

\begin{tabular}{|c|c|c|c|c|c|}
\hline & & \multicolumn{4}{|c|}{ Sodium chloride concentration } \\
\hline & & $\mathbf{0 \%}$ & $1.5 \%$ & $3 \%$ & $4.5 \%$ \\
\hline \multicolumn{2}{|l|}{ Species } & \multicolumn{4}{|c|}{ Reduction [\%] } \\
\hline Enterococcus faecalis & 201701784.1.K & $27.65^{*}$ & $18.90 *$ & $31.30 *$ & 4.80 \\
\hline Enterococcus faecium & VF21.2-1122.3.K & $33.44 *$ & $32.79 *$ & $41.00 *$ & 5.51 \\
\hline Enterococcus durans & 201702044.1.K & $31.96^{*}$ & 15.04 & $12.76^{*}$ & 6.57 \\
\hline Staphylococcus equorum & 201701784.3.B & $72.80 *$ & $49.16 *$ & 9.39 & 4.17 \\
\hline Staphylococcus simulans & 201702044.2.B & $99.93 *$ & $82.56^{*}$ & $75.75^{*}$ & 4.94 \\
\hline Staphylococcus xylosus & 201702052.2.B & $99.93 *$ & $53.73 *$ & $40.85 *$ & 9.23 \\
\hline Lactococcus lactis & P.6.2.M & $50.26^{*}$ & $69.12 *$ & 6.25 & -1.37 \\
\hline Lactobacillus parabuchneri & P.12.3.M & $41.44 *$ & $37.65 *$ & 7.57 & 4.45 \\
\hline
\end{tabular}

${ }^{*} P<0.05$

At a sodium chloride concentration of $4.5 \%$, the ability of Lb. plantarum JA-1199 to reduce tyramine accumulation significantly decreased in all eight micro-cheese model setups and showed a maximum of $9.23 \%$ of reduction at S. xylosus 201702052.2.B with tyramine concentrations of $520.77 \mathrm{mg} \mathrm{L}^{-1}$ in combination with $\mathrm{Lb}$. plantarum JA-1199 and $573.75 \mathrm{mg} \mathrm{L}^{-1}$ without, respectively. Moreover, at $4.5 \%$ sodium chloride, L. lactis P.6.2.M was even able to produce a minimal amount more tyramine in combination with $L b$. plantarum JA-1199 compared to no added $L b$. plantarum JA-1199. In combination with $S$. simulans 201702044.2.B or S. xylosus 201702052.B, Lb. plantarum JA1199 showed the highest reduction efficiency of tyramine accumulation of $99.93 \%$ at $0 \%$ of sodium chloride. In combination with $S$. simulans 201702044.2.B, tyramine concentrations of 'below detection limit', $61.00 \mathrm{mg} \mathrm{L}^{-1}$ and $34.86 \mathrm{mg} \mathrm{L}^{-1}$ in $0 \%, 1.5 \%$ and $3 \%$ sodium chloride were detected and without $\mathrm{Lb}$. plantarum JA-1199 concentrations of $72.12 \mathrm{mg} \mathrm{L}^{-1}, 349.82 \mathrm{mg} \mathrm{L}^{-1}$ and $143.73 \mathrm{mg} \mathrm{L}^{-1}$, respectively. In combination with $S$. xylosus 201702052.2.B, tyramine concentrations of 'below detection limit', $305.01 \mathrm{mg} \mathrm{L}^{-1}$, and $191.01 \mathrm{mg} \mathrm{L}^{-1}$ in $0 \%, 1.5 \%$ and $3 \%$ sodium chloride could be detected and without $L b$. plantarum JA-1199 concentrations of $67.91 \mathrm{mg} \mathrm{L}^{-1}, 659.17 \mathrm{mg} \mathrm{L}^{-1}$ and $322.92 \mathrm{mg} \mathrm{L}^{-1}$, respectively, which is a reduction of tyramine accumulation of $9.93 \%, 82.56 \%$ and $75.75 \%$ for $S$. simulans 201702044.2 .B and $99.93 \%, 53.73 \%$, and $40.85 \%$ for S. xylosus 201702052.2.B, respectively. As without $L b$. plantarum JA-1199, E. faecalis 201701784.1.K also produced a relatively stable and high tyramine concentration in combination with $L b$. plantarum JA-1199 in all four tested sodium chloride concentrations. Moreover, Lb. plantarum JA-1199 showed a lower activity, with a maximum reduction of tyramine accumulation of $31.30 \%$ in a micro-cheese
In order to confirm the previous data of tyrosine degradation of $L b$. plantarum JA-1199 and to verify its ability to degrade tyrosine in a micro-cheese model, $L b$. plantarum JA-1199 was incubated with and without $2.5 \mathrm{mM}$ tyrosine at different sodium chloride concentrations $(0 \%, 1.5 \%, 3 \%$ and $4.5 \%)$. It could be shown that $L b$. plantarum JA-1199 has the ability to reduce tyrosine concentration up to $98.66 \%$, independent of sodium chloride concentration (data not shown). However, a small amount of tyramine was found. Tyramine concentration increased with sodium chloride concentration and had a maximum concentration at $4.5 \%$ sodium chloride with a value of $57.08 \mathrm{mg} \mathrm{L}^{-1}$. The incubation of $L b$. plantarum JA-1199 without tyrosine at different sodium chloride concentrations showed negligibly small values with a maximum of $14.46 \mathrm{mg} \mathrm{L}^{-1}$ (4.5\% sodium chloride after 7 days of ripening time) for tyrosine and $8.66 \mathrm{mg} \mathrm{L}^{-1}$ (3\% sodium chloride after 7 days of ripening time) for tyramine, respectively. All tyramine concentrations prior to incubation were below the detection limit of $0.05 \mathrm{mg} \mathrm{L}^{-1}$. Furthermore, it was verified that Lb. plantarum JA-1199 does not inhibit per se the growth of tyramine-producing bacteria, and therefore less tyramine would be produced. Tyramine-producing bacteria were thus plated without and in combination with Lb. plantarum JA-1199 on the corresponding semi-selective media-agar and incubated. It could be confirmed that the growth of the tyramine-producing strain in combination with $L b$. plantarum JA-1199 was in the same log count as in growth without, respectively (data not shown).

To confirm that tyrosine concentrations in the micro-cheese model remain stable during incubation without bacterial strains, micro-cheese models were incubated with initial $2.5 \mathrm{mM}$ tyrosine at four different sodium chloride concentrations $(0 \%, 1.5 \%, 3 \%$ and $4.5 \%$ ). It was shown that the tyrosine concentration remained 
Table 3. Production of tyramine in $\mathrm{mg} \mathrm{L}^{-1}$ by eight different tyramine-producing bacterial strains in combination with $\mathrm{Lb}$. plantarum $\mathrm{JA} 1199$ in microcheese without tyrosine at four different sodium chloride concentrations

\begin{tabular}{|c|c|c|c|c|c|}
\hline & & \multicolumn{4}{|c|}{ Sodium chloride concentration } \\
\hline \multirow{2}{*}{\multicolumn{2}{|c|}{ Species }} & $0 \%$ & $1.5 \%$ & $3 \%$ & $4.5 \%$ \\
\hline & & \multicolumn{4}{|c|}{ Tyramine production $\left[\mathrm{mg} \mathrm{L}^{-1}\right]$} \\
\hline Enterococcus faecalis & 201701784.1.K & 18.98 & 9.14 & 11.60 & 9.81 \\
\hline Enterococcus faecium & VF21.2-1122.3.K & 5.24 & 9.80 & 12.50 & 13.76 \\
\hline Enterococcus durans & 201702044.1.K & 6.02 & 7.51 & 11.71 & 9.81 \\
\hline Staphylococcus equorum & 201701784.3.B & b.d. & 2.09 & 6.35 & 4.25 \\
\hline Staphylococcus simulans & 201702044.2.B & b.d. & b.d. & b.d. & 3.60 \\
\hline Staphylococcus xylosus & 201702052.2.B & b.d. & 2.32 & 2.56 & 2.79 \\
\hline Lactococcus lactis & P.6.2.M & 6.15 & 4.21 & 11.98 & 10.90 \\
\hline Lactobacillus parabuchneri & P.12.3.M & 7.08 & 8.87 & 12.26 & 10.94 \\
\hline
\end{tabular}

b.d.: below detection limit

stable with a marginal variation of $3.95 \%$ at $0 \%, 1.19 \%$ at $1.5 \%$ and $1.26 \%$ at $3.0 \%$ sodium chloride, respectively. At a sodium chloride concentration of $4.5 \%$, a slight increase of tyrosine of $12.93 \%$ from $448.61 \mathrm{mg} \mathrm{L}^{-1}$ up to $506.62 \mathrm{mg} \mathrm{L}^{-1}$ was observed (data not shown). Importantly, all tyrosine and tyramine concentrations before and all tyrosine concentrations after ripening without tyrosine addition at different sodium chloride concentrations were below the detection limit of $0.13 \mathrm{mg} \mathrm{L}^{-1}$ and $0.05 \mathrm{mg} \mathrm{L}^{-1}$, respectively. After 7 days of ripening, only very low tyramine concentrations of $7.33 \mathrm{mg} \mathrm{L}^{-1}$ at $0 \%$ sodium chloride, $5.84 \mathrm{mg} \mathrm{L}^{-1}$ at $1.5 \%$ sodium chloride, $7.42 \mathrm{mg} \mathrm{L}^{-1}$ at $3 \%$ sodium chloride and $2.34 \mathrm{mg} \mathrm{L}^{-1}$ at $4.5 \%$ sodium chloride were detected (data not shown).

\section{Discussion}

The accumulation of BAs in fermented foods is a matter of public health concern, and it is important to know, which factors affecting the production of BAs and how to prevent or reduce the accumulation in fermented foods. ${ }^{[47]}$

It is known that mono- and diaminooxidase catalyse the detoxifying oxidation of BAs in the gastrointestinal tract of higher organisms. ${ }^{[48]}$ Furthermore, amino oxidases were identified in Arthrobacterkristallopoes, Bacillus amyloliquefaciens, Candida boidinii, Klebsiella aerogenes, Lactobacillus and Pediococcus spp. Micrococcus rubens, Rhodococcus erythropolis, Sarcina lutea and Staphylococcus carnosus. Although the exact mechanism of action is still unclear, amine-degrading bacteria are used to reduce BAs in food. ${ }^{[43,49-54]}$ The amine-degrading activity of the lactic acid bacteria $\mathrm{Lb}$. plantarum $\mathrm{J} 16$ was attributed to a laccase (EC 1.10.3.2). ${ }^{[55]}$ This enzyme belongs to the multicopper oxidase superfamily and their catalytic centres contain four reactive copper atoms, giving them a characteristic blue colour. ${ }^{[56}$ Furthermore, it is known that aminotransferases catalyse the transfer of an amino group from the amino acid acting as a substrate to an acceptor oxo acid. ${ }^{[57]}$ Therefore, aminotransferases play an essential role in the metabolism of amino acids as the resulting product 4-hydroxy-phenylpyruvate can be further metabolised to succinate and finally used as an energy source in the Citrate cycle as an energy gain. ${ }^{[58]}$ In this study, the in silico screening of Lb. plantarum JA-1199 identified a multicopper oxidase as well as a histidinol-phosphate transaminase (EC 2.6.1.9). The biological roles of multicopper oxidases in bacteria are poorly elucidated, but it is known that laccases oxidise phenols by a radical-generating reaction mechanism. ${ }^{[59,60]}$ Furthermore, in this study, the use of tyrosine by $L b$. plantarum JA-1199 was phenotypically demonstrated using a Biolog microarray assay and through incubation in $\mathrm{mCDM}$ and milk both containing tyrosine. Tyrosine metabolism contains various enzymatic pathways via tyrosinases and laccases to L-dopa or via aminotransferases to 4-hydroxy-phenylpyruvate, and it may be hypothesised that the capacity of $L b$. plantarum JA-1199 and its histidinol-phosphate transaminase (EC 2.6.1.9), which shows homology to the aspartate aminotransferase and tyrosine aminotransferase, namely aspartate aminotransferase (EC 2.6.1.1) and tyrosine aminotransferase (EC 2.6.1.5) ${ }^{[57]}$ to use tyrosine are based on this metabolism.

Previous studies reported that sodium chloride has an influence on decarboxylase activity and thus on BA accumulation in fermented foods. ${ }^{[61]}$ A higher sodium chloride concentration increases the amount of BAs produced by increased enzyme activity and the expression of genes involved in BA production. ${ }^{[61-63]}$ In this study, the influence of sodium chloride on tyramine production could be confirmed. However, a high variability on tyramine concentration from different bacteria and their response to different sodium chloride concentrations was observed. Thus, it could be demonstrated that representatives of different tyramine-producing bacterial species react differently to an increased sodium chloride concentration.

Because of its use of the substrate tyrosine as a nitrogen source, in this study, Lb. plantarum JA-1199 was successfully used to reduce tyramine accumulation in different environments. Compared to the results without $L b$. plantarum JA-1199, the micro-cheeses containing this strain showed significantly lower amounts of tyramine with a reduction of up to $99 \%$ in co-incubation with S. simulans 201702044.2.B and S. xylosus 201702052.2.B. However, this study also showed a drastic decrease in the ability of Lb. plantarum JA-1199 to reduce tyramine accumulation as a response to an increase in sodium chloride concentration up to $4.5 \%$. This effect can be confirmed by the potential role of osmoprotection of decarboxylase genes as a part of complex metabolic responses in the presence of stress conditions. The TDC cluster in tyramineproducing bacteria contains a $\mathrm{Na}^{+} / \mathrm{H}^{+}$transporter encoding gene and sodium ions $\left(\mathrm{Na}^{+}\right)$are known to be involved in the regulation of intracellular $\mathrm{pH}$ and are important in sodium/proton antiport systems by exchange with $\mathrm{H}^{+}$ions that are removed from the cell. Therefore, sodium ions play an essential role in the tyrosine decarboxylation pathway and may explain the higher tyramine production at a higher sodium chloride concentration as proposed 
by other authors. ${ }^{[61,64,65]}$ However, since salt is used in fermented food products to prevent spoilage and food poisoning, ${ }^{[22]}$ reducing salt concentration in cheese is not an option to reduce the tyramine content in cheese. The minimal concentration of tyramine found in the micro-cheese model incubated only with $L$ b. plantarum JA1199, as well as in the control can be explained by the fact that milk may already contain tyramine at low dosages. ${ }^{[15,21]}$

Lb. plantarum is a common member of the non-starter lactic acid bacteria in cheeses and given the concerns over the presence of tyramine concentrations in different cheeses, this study showed a feasible strategy for decreasing tyramine accumulation and therefore increasing the safety level of fermented foods by application of a food-grade biocontrol strain.

\section{Acknowledgements}

We are grateful to the Swiss Federal Food Safety and Veterinary Office (FSVO) for funding this study. The authors also extended their acknowledgements to Dr. Ueli von Ah, and the whole Agroscope team, for their support. We would also like to thank Iris Häsuermann, Serafina Plüss and Shera Ly for their work during their Bachelor thesis and Simon Gürber for his work during his Master thesis.

\section{Conflict of interest}

The authors declare that they have no conflict of interests.

Received: February 24, 2020

[1] D. M. Linares, M. Cruz, Martín, V. Ladero, M. A. Alvarez, M. Fernández, Crit. Rev. Food Sci. Nutr. 2011, 8398, 94.

[2] M. Fernández, D. M. Linares, A. Rodríguez, M. A. Alvarez, Appl. Microbiol. Biotechnol. 2007, 73, 1400.

[3] A. Herrero-Fresno, N. Martínez, E. Sánchez-Llana, M. Díaz, M. Fernández, M. C. Martin, V. Ladero, M. A. Alvarez, Int. J. Food Microbiol. 2012, 157, 297.

[4] S. H. Lee, J. Y. Jung, C. O. Jeon, J. Food Sci. 2015, 80, 1871.

[5] V. Moreno-Arribas, FEMS Microbiol. Lett. 2002, 195, 103.

[6] J. S. Moon, S. Y. Kim, K. J. Cho, S. J. Yang, G. M. Yoon, H. J. Eom, N. S. Han, J. Microbiol. 2013, 51, 881.

[7] G. Suzzi, F. Gardini, Int. J. Food Microbiol. 2003, 88, 41.

[8] E. Boonstra, R. de Kleijn, L. S. Colzato, A. Alkemade, B. U. Forstmann, S. Nieuwenhuis, Front. Psychol. 2015, 6, 6.

[9] C. V. Borges, M. A. F. Belin, E. P. Amorim, I. O. Minatel, G. C. Monteiro, H. A Gomez Gomez, G. R. S. Monar, G. P. P. Lima, Food Chem. 2019, 298, 125020

[10] P. Kalač, Food Chem. 2014, 161, 27.

[11] R. K. Dismukes, A. V. Rake, Psychopharmacologia 1973, 29, 91.

[12] V. Ladero, M. Fernández, M. Calles-Enríquez, E. Sánchez-Llana, E. Cañedo, M. C. Martín, M. A. Alvarez, Food Microbiol. 2012, 30, 132.

[13] K. J. Broadley, Pharmacol. Ther. 2010, 125, 363.

[14] E. De Mey, K. De Klerck, H. De Maere, L. Dewulf, G. Derdelinckx, M. C. Peeters, I. Fraeye, Y. Vander Heyden, H. Paelinck, Meat Sci. 2014, 96, 821.

[15] EFSA, EFSA J. 2011, 9, 2393.

[16] P. Combarros-Fuertes, D. Fernández, R. Arenas, I. Diezhandino, M. E. Tornadijo, J. M. Fresno, J. Sci. Food Agric. 2015, 96, 295.

[17] EFSA, EFSA J. 2005, 50, 1.

[18] M. L. Latorre-Moratalla, S. Bover-Cid, M. T. Veciana-Nogués, M. C. VidalCarou, Front. Microbiol. 2012, 3, 1.

[19] M. Fernández, A. Belén Flórez, D. M. Linares, B. Mayo, M. A. Alvarez, J. Dairy Res. 2006, 73, 318.

[20] F. Galgano, G. Suzzi, F. Favati, M. Caruso, M. Martuscelli, F. Gardini, G. Salzano, Int. J. Food Sci. Technol. 2001, 36, 153.

[21] S. Novella-Rodríguez, M. T. Veciana-Nogués, A. X. Roig-Sagués, A. J. Trujillo-Mesa, M. C. Vidal-Carou, J. Dairy Res. 2004, 71, 245.

[22] D. M. Linares, B. Del Río, V. Ladero, N. Martínez, M. Fernández, M. Cruz Martín, Front. Microbiol. 2012, 3, 1.

[23] S. Torriani, V. Gatto, S. Sembeni, R. Tofalo, G. Suzzi, N. Belletti, F. Gardini, S. Bover-Cid, J. Food Prot. 2016, 71, 93.

[24] M. Coton, M. Fernández, H. Trip, V. Ladero, N. L. Mulder, J. S. Lolkema, M. A. Alvarez, E. Coton, Microbiology 2011, 157, 1841.

[25] Á. Marcobal, B. de las Rivas, J. M. Landete, L. Tabera, R. Muñoz, Crit. Rev. Food Sci. Nutr. 2012, 52, 448.

[26] M. Fernández, D. M. Linares, M. A. Alvarez, J. Food Prot. 2004, 67, 2521.

[27] M. L. Mohedano, P. López, G. Spano, P. Russo, in 'Advances in Fermented Foods and Beverages: Improving Quality, Technologies and Health Benefits', 1 ed., Ed. W. Holzapfel, Elsevier Ltd., 2015, pp. 273.

[28] S. Bover-Cid, M. Hugas, M. Izquierdo-Pulido, M. C. Vidal-Carou, Int. J. Food Microbiol. 2001, 66, 185
[29] J. Emborg, P. Dalgaard, Int. J. Food Microbiol. 2008, 128, 234.

[30] J. Emborg, P. Dalgaard, Int. J. Food Microbiol. 2008, 128, 226.

[31] F. Gardini, M. Martuscelli, M. C. Caruso, F. Galgano, M. A. Crudele, F. Favati, M. E. Guerzoni, G. Suzzi, Int. J. Food Microbiol. 2001, 64, 105.

[32] F. Liu, X. Wang, L. Du, D. Wang, Y. Zhu, Z. Geng, X. Xu, W. Xu, J. Food Prot. 2015, 78, 940.

[33] Y. Ardö, P. L. H. McSweeney, A. A. A. Magboul, V. K. Upadhyay, P. F. Fox, in 'Cheese: Chemistry, Physics and Microbiology', 3 ed., Eds. P. F. Fox, P. L. H. McSweeney, T. M. Cogan, T. P. Guinee, Elsevier Ltd, 2017, pp. 445.

[34] A. Naila, S. Flint, G. Fletcher, P. Bremer, G. Meerdink, J. Food Sci. 2010, 75, 139.

[35] M. Fernández, D. M. Linares, B. Del Río, V. Ladero, M. A. Alvarez, J. Dairy Res. 2007, 74, 276.

[36] S. Novella-Rodríguez, M. T. Veciana-Nogués, A. X. Roig-Sagués, A. J. Trujillo-Mesa, M. C. Vidal-Carou, J. Dairy Sci. 2010, 85, 2471.

[37] R. Guarcello, M. De Angelis, L. Settanni, S. Formiglio, R. Gaglio, F. Minervini, G. Moschetti, M. Gobbetti, Appl. Environ. Microbiol. 2016, 82, 6870 .

[38] S. Jalkanen, M. Salmi, EMBO J. 2001, 20, 3893.

[39] P. J. Hoegger, S. Kilaru, T. Y. James, J. R. Thacker, U. Kües, FEBS J. 2006, 273, 2308.

[40] K. U. Zaidi, A. S. Ali, S. A. Ali, I. Naaz, Biochem. Res. Int. 2014, 2014, 1.

[41] L. Li, X. Wen, Z. Wen, S. Chen, L. Wang, X. Wei, Front. Microbiol. 2018, 9 , 1.

[42] S. Callejón, R. Sendra, S. Ferrer, I. Pardo, Appl. Microbiol. Biotechnol. 2014, $98,185$.

[43] R. A. Cooper, FEMS Microbiol. Lett. 1997, 146, 85

[44] D. Hasegawa, K. Kito, T. Maeda, V. Rai, S. Cha-um, Y. Tanaka, M. Fukaya, T. Takabe, Protoplasma 2019, 256, 1727.

[45] Dionex, Dionex 2007, 183, 1.

[46] M. Garriga, M. Aymerich, M. Costa, J. Monfort, M. Hugas, Food Microbiol. 2002, 19, 509 .

[47] Y. Özogul, F. Özogul, in 'Biogenic Amines in Food: Analysis, Occurrence and Toxicity', 1 ed., Eds. B. Saad, R. Tofalo, Royal Society Of Chemistry, $\mathbf{2 0 2 0}$, pp. 1.

[48] M. B. H. Youdim, D. Edmondson, K. F. Tipton, Nat. Rev. Neurosci. 2006, 7, 295.

[49] O. V. Yagodina, E. B. Nikol'skaya, A. E. Khovanskikh, B. N. Kormilitsyn, J. Evol. Biochem. Physiol. 2002, 38, 251.

[50] H. Ota, H. Tamezane, Y. Sasano, E. Hokazono, Y. Yasuda, S. I. Sakasegawa, S. Imamura, T. Tamura, S. Osawa, Biosci. Biotechnol. Biochem. 2008, 72, 2732.

[51] E. W. Van Hellemond, M. Van Dijk, D. P. H. M. Heuts, D. B. Janssen, M. W. Fraaije, Appl. Microbiol. Biotechnol. 2008, 78, 455.

[52] V. Capozzi, P. Russo, V. Ladero, M. Fernández, D. Fiocco, M. A. Alvarez, F. Grieco, G. Spano, Front. Microbiol. 2012, 3, 1.

[53] M. Z. Zaman, F. Abu Bakar, S. Jinap, J. Bakar, Int. J. Food Microbiol. 2011, $145,84$.

[54] A. García-Ruiz, E. M. González-Rompinelli, B. Bartolomé, M. V. MorenoArribas, Int. J. Food Microbiol. 2011, 148, 115.

[55] S. Callejón, R. Sendra, S. Ferrer, I. Pardo, Appl. Microbiol. Biotechnol. 2016, 100, 3113.

[56] R. Reiss, J. Ihssen, M. Richter, E. Eichhorn, B. Schilling, L. Thöny-Meyer, PLoS One 2013, 8, e65633.

[57] P. K. Mehta, T. I. Hale, P. Christen, Eur. J. Biochem. 1989, 186, 249.

[58] Tyrosine metabolism, https://www.kegg.jp/dbget-bin/www_bget?map00350.

[59] P. Sharma, R. Goel, N. Capalash, World J. Microbiol. Biotechnol. 2007, 23, 823.

[60] H. Claus, Arch. Microbiol. 2003, 179, 145.

[61] L. Buáková, F. Buška, E. Pollaková, T. Podešvová, V. Dráb, Int. J. Food Microbiol. 2011, 147, 112.

[62] V. Ladero, D. M. Linares, M. Pérez, B. del Rio, M. Fernández, M. A. Alvarez, in 'Microbial Toxins in Dairy Products', 1 ed., Ed. A. Y. Tamine, Wiley, 2016, pp. 94.

[63] L. Buňková, F. Buňka, V. Dráb, S. Kráčmar, V. Kubáň, Eur. Food Res. Technol. 2012, 234, 973

[64] G. Tabanelli, S. Torriani, F. Rossi, L. Rizzotti, F. Gardini, J. Food Sci. 2012, $77,231$.

[65] C. I. Pereira, D. Matos, M. V. San Romão, M. T. Barreto Crespo, Appl. Environ. Microbiol. 2009, 75, 345.

\section{License and Terms}

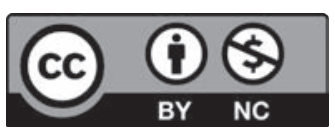

This is an Open Access article under the terms of the Creative Commons Attribution License CC BY_NC 4.0. The material may not be used for commercial purposes.

The license is subject to the CHIMIA terms and conditions: (http:// chimia.ch/component/sppagebuilder/?view=page\&id=12).

The definitive version of this article is the electronic one that can be found at doi:10.2533/chimia.2020.391 